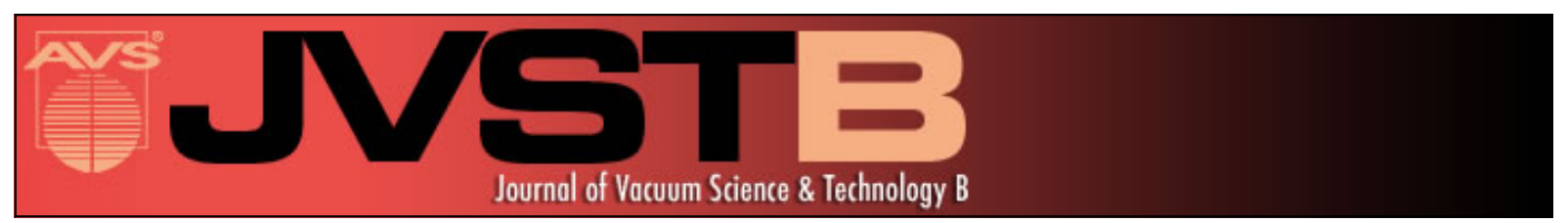

\title{
In-situ visualization of local magnetic fields using low-energy electron beam in scanning electron microscope
}

Konomi Yoshida, Katsuhisa Murakami, and Jun-ichi Fujita

Citation: Journal of Vacuum Science \& Technology B 32, 06FC02 (2014); doi: 10.1116/1.4897504

View online: http://dx.doi.org/10.1116/1.4897504

View Table of Contents: http://scitation.aip.org/content/avs/journal/jvstb/32/6?ver=pdfcov

Published by the AVS: Science \& Technology of Materials, Interfaces, and Processing

\section{Articles you may be interested in}

In situ nanomechanical testing in focused ion beam and scanning electron microscopes

Rev. Sci. Instrum. 82, 063901 (2011); 10.1063/1.3595423

A design for a subminiature, low energy scanning electron microscope with atomic resolution

J. Appl. Phys. 105, 014702 (2009); 10.1063/1.3058602

In situ monitoring of material processing by a pulsed laser beam coupled via a lensed fiber into a scanning electron microscope

J. Vac. Sci. Technol. A 26, 1432 (2008); 10.1116/1.2987946

Magnetic axial field measurements on a high resolution miniature scanning electron microscope

Rev. Sci. Instrum. 71, 1712 (2000); 10.1063/1.1150525

Electron microscopy and nanolithography with an integrated low-energy electron beam

Appl. Phys. Lett. 75, 1807 (1999); 10.1063/1.124827

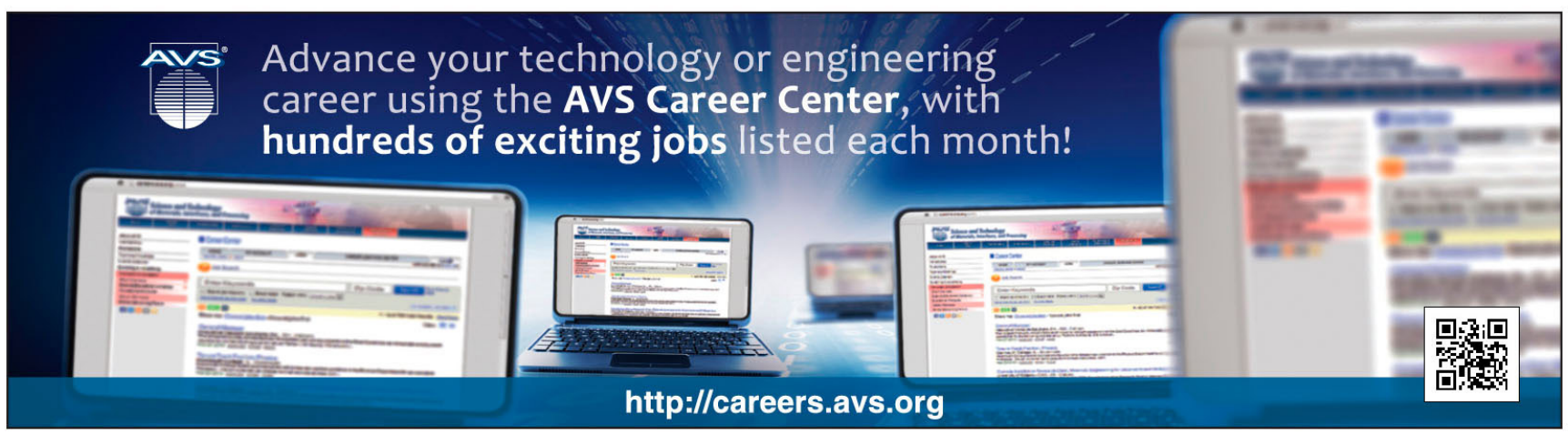




\title{
In-situ visualization of local magnetic fields using low-energy electron beam in scanning electron microscope
}

\author{
Konomi Yoshida, Katsuhisa Murakami, and Jun-ichi Fujita ${ }^{a)}$ \\ Institute of Applied Physics, University of Tsukuba, 1-1-1 Tennodai, Tsukuba, Ibaraki 305-8571, \\ Japan and Tsukuba Research Center for Interdisciplinary Materials Science (TIMS), University of Tsukuba, \\ 1-1-1 Tennodai, Tsukuba, Ibaraki 305-8571, Japan
}

(Received 1 July 2014; accepted 29 September 2014; published 9 October 2014)

\begin{abstract}
This study demonstrates clear visualization of the magnetic field around a two-dimensional magnetic material using the Lorentz deflection of a primary electron beam with low accelerating voltage. A horizontal or vertical grid, positioned downstream of the specimen, appears on the scanning microscope image where the field of view shows both the specimen edge and the grid below. An observed deformation in the grid shape is strongly correlated with the local magnetic field and is related to the beam deflection induced by the Lorentz force. A detection sensitivity of $\sim 0.5 \mathrm{G}$ is achieved using a primary electron beam with an energy of $1 \mathrm{keV}$. (C) 2014 American Vacuum Society. [http://dx.doi.org/10.1116/1.4897504]
\end{abstract}

\section{INTRODUCTION}

Visualization of local magnetic fields in two-dimensional magnetic materials is imperative for a better understanding of spintronic and electronic behavior. Localization of the spin, flow, and spin-wave interference ${ }^{1-3}$ plays an important role in determining the device functionality. ${ }^{4-6}$ However, the magnetic flux of a single spin is quite small, wherein the magnetic dipole moment of a single spin is $\mu_{0} \mu_{B}=1.2 \times 10^{-29} \mathrm{~Wb} \cdot \mathrm{m}$, and the magnetic field generated by that single spin at a distance $1 \mathrm{~nm}$ along the dipole axis is $18 \mathrm{G}$.

Magnetic force microscopy (MFM) is a powerful candidate for visualizing the local magnetization. ${ }^{7-10}$ The MFM method relies upon detecting the attractive and/or repulsive forces that affect the magnetized top layer of a probing tip. The sensitivity to the local magnetization strongly depends upon the distance between the tip and the magnetized surface; therefore, a technique called interleave scanning ${ }^{11}$ is used to detect the contrast of the magnetic force on the specimen by keeping the separation gap constant. In this way, MFM enables visualization of the distribution of magnetization on a material rather than the distribution of the magnetic field.

Electron beam holography ${ }^{12-19}$ is a popular and conventional technique that can be used to visualize local magnetic fields. In this technique, the coherent electron beam is divided into a reference and object wave using an electron biprism ${ }^{13}$ prior to irradiating the specimen. The electron waves that pass through the magnetic field or the magnetized material interfere with the reference waves, thereby creating interference fringes on the screen. The phase shift, $\Delta \phi(x)$, of an electron that has passed through the magnetized specimen relative to an electron that has passed through only a vacuum is given by

$$
\Delta \phi(x)=-\frac{e}{\hbar} \iint B_{y}(x, z) d x d z,
$$

where $z$ is the incident beam direction, $x$ is the direction in the plane of the specimen, $B_{y}$ is the magnetic flux density in

a)Electronic mail: fujita@bk.tsukuba.ac.jp the direction perpendicular to both $x$ and $z, e$ is the elementary charge, and $\hbar$ is Planck's constant. When the electron passes through a uniformly magnetized material that has a constant thickness $l_{z}$, the phase shift can be reduced to $\Delta \phi=-e B l_{z} / \hbar$. The phase shift can be also rewritten as

$$
\Delta \phi=-(2 \pi / \lambda) \frac{e B l_{z}}{m_{e} v_{0}},
$$

using de Broglie's wave length $\lambda$ for the electron velocity $v_{0}$ and electron mass $m_{e}$. Here, the horizontal velocity $v_{h}$, which the electron develops during the passage through the magnetic field, is given by

$$
v_{h}=-\frac{e v_{0} B}{m_{e}}\left(\frac{l_{z}}{v_{0}}\right),
$$

with the consideration that the transit time in the field is $l_{z} /$ $v_{0}$. The deflection angle $\theta$ can be defined by

$$
\tan \theta=\frac{v_{h}}{v_{0}}=\frac{e B l}{m_{e} v_{0}} .
$$

Therefore, electron holography can be said to detect the phase shift $\Delta \phi=(2 \pi / \lambda) \tan \theta$ created by the interference of the deflected electron wave with a reference wave that has been slightly refracted by the electron biprism.

Electron holography can sensitively detect phase shifts of $\pi / 100$ by combining digital image processing and visualization of the magnetization of nanosized particles. Typically, this method allows for the clear visualization of coercive fields $\sim 300-450$ Oe in size from a 30-nm-thick magnetic crystal in a biocell. ${ }^{16,17}$

Another widely used method for visualizing magnetization is the Lorentz microscope, ${ }^{20,21}$ which uses the deflection of the electron beam induced by the Lorentz force. Deflection of the electron beam by a magnetized material generates a dark contrast against the nondeflected projection. The position of the interference-induced dark contrast varies depending upon the focus depth, and therefore, the local magnetic field can be evaluated by measuring the position 
shift of the deflected image. The deflection angle is inversely proportional to the voltage used to accelerate the beam, so larger deflection angles can be obtained with lower accelerating voltages. ${ }^{19}$ However, higher image precision requires a higher accelerating voltage, which produces a trade-off with the detection sensitivity.

We have previously demonstrated that the local electric field around a sharp apex point of a biasing probe can be clearly visualized by using scanning electron microscope (SEM). ${ }^{22-25}$ Here, we demonstrate that the magnetic field distribution around a two-dimensional magnetized material can also be visualized using the beam deflection of electrons under low acceleration.

\section{EXPERIMENT}

The deflection of the primary electron beam is detected by a gold grid placed just beneath the specimen, as shown in the schematic of Fig. 1. The primary electron beam travels straight past the specimen if the specimen is not magnetized, following the path marked with dashed trace lines in Fig. 1. Secondary electrons generated at the gold grid create somewhat diffuse shadowy lines at the back of the SEM image, where the shadowy line in this case represents the shape of the gold grid. By contrast, with a magnetized specimen, the beam is deflected by the local magnetic field, following the path marked by the solid line in Fig. 1. The deflection of the primary beam strongly deforms the shadowy lines, representing the magnetic field distribution, and the local magnetic field is calculated using the deflection angle.
In this experiment, we used a Hitachi S4800 SEM whose normal operation mode was the semi-in-lens mode. In this mode, some magnetic field lines stray from the objective lens, creating a stray field that works to minimize the focusing aberration. However, this stray field is relatively high, reaching several thousands of Gauss, which sometimes destroyed the intrinsic field distribution of the specimen. Thus, magnetic field visualization was accomplished using the low magnification mode of the SEM in which the outermost objective lens for high precision focusing was turned off, thereby creating a space that was relatively free of magnetic fields around the specimen. This low-magnification mode created another advantage for field visualization in that the depth of the focus is rather large and is inevitably linked to the deep position of the focusing lens in the low-magnification mode and, therefore, beam focusing was sustained at the grid position to enable recognition of the grid shape.

Typical accelerating voltages were in the range of $1-10 \mathrm{keV}$, and the back pressure was held below $10^{-3} \mathrm{~Pa}$. The periodicity of the gold grid was $10 \mu \mathrm{m}$, and each grid line was $\sim 1.5 \mu \mathrm{m}$ wide. Horizontal and vertical grids were used in this study. The horizontal grid was used to detect the horizontal component of the magnetic field, because vertical deflection is given by the cross product of the electron velocity and the horizontal component of the magnetic field. By the same reasoning, the vertical grid was used to detect the vertical component of the magnetic field.

A gold grid array was placed $\sim 22 \mathrm{~mm}$ downstream from the specimen, and secondary electrons produced by the deflected primary electron beam impinging upon the grid

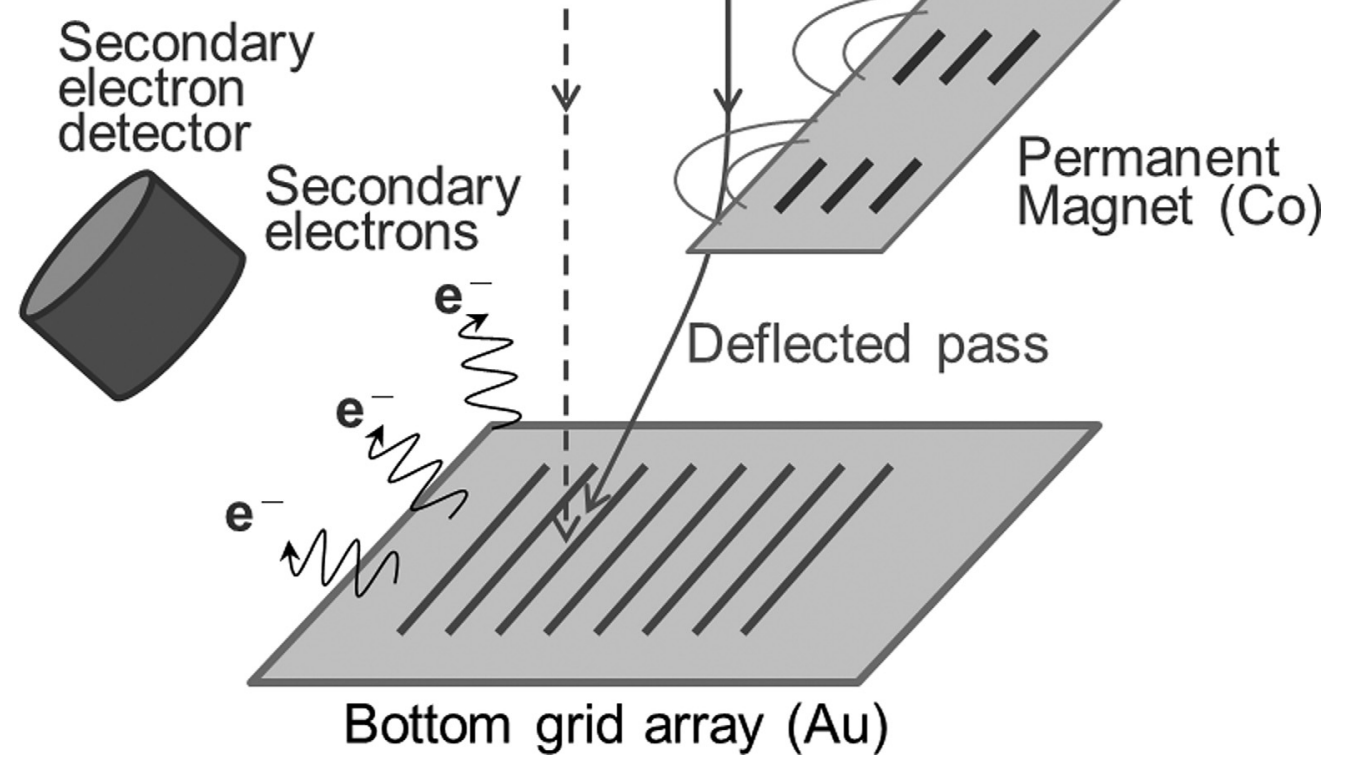

FIG. 1. Schematic of the magnetic field visualization setup. 
array generated bright contour lines that were simultaneously superimposed in the SEM image. The model specimen used in this study was a cobalt magnetic dot array constructed from rectangular-shaped dots each $10 \mu \mathrm{m}$ long, $2 \mu \mathrm{m}$ wide, and $200 \mathrm{~nm}$ thick. The periodicity of the dot array was $30 \mu \mathrm{m}$ in the horizontal direction and $15 \mu \mathrm{m}$ in the vertical direction.

\section{RESULTS}

Figure 2 shows a typical example of the field images obtained using the horizontal and vertical grid, and the corresponding schematic deflection schemes. The magnetic field distribution for this simple array of dots can be easily resolved using the finite element method (FEM) [Fig. 2(e)] without performing this sensitive experiment. However, a precise and real-time visualization remains imperative for a better understanding of the spintronic device application, and analytical results could serve well as an evaluation of the possibilities of this field visualization technique. At point A in Fig. 2(c), the electron beam arrives normally from the top and goes into the picture plane, though the current is in the opposite direction because of the negative sign of the electron charge. The local magnetic field around point $\mathrm{A}$ is directed diagonally upwards to the right [gray arrow in Fig. 2(c)], so the Lorentz force deflects the beam toward $A^{\prime}$ [black arrow in Fig. 2(c)]. In this example, the grid line does not exist directly beneath point $\mathrm{A}$, but the deflected beam that reaches point $\mathrm{A}^{\prime}$ is able to hit the grid line. The secondary electrons that are emitted from the grid generate a deformed shadow line seen in the SEM image that makes it appear that the grid exists at point A. By the same token, the electron beam at point $\mathrm{B}$ will be deflected to $\mathrm{B}^{\prime}$, and the resulting grid shadow will be strongly bent and mirrorsymmetric around the center of the cobalt dot rectangular array. The maximal field intensity is the $y$-component just above the center of the rectangle, which causes maximal beam deflection. Therefore, the obtained shadow is hidden by the specimen surface with the large positional shift to the bottom.

The deformation obtained using the vertical grid follows a similar form. In Fig. 2(d), the primary beam that is deflected toward $\mathrm{C}^{\prime}$ creates a left-hand convergence, and the beam that reaches $\mathrm{D}^{\prime}$ creates a right-hand convergence. Here, the $y$-component of the magnetic field along the central axis of the rectangle is exactly zero, and thus, the grid shadow at any point along the central axis is straight and experiences no deformation.
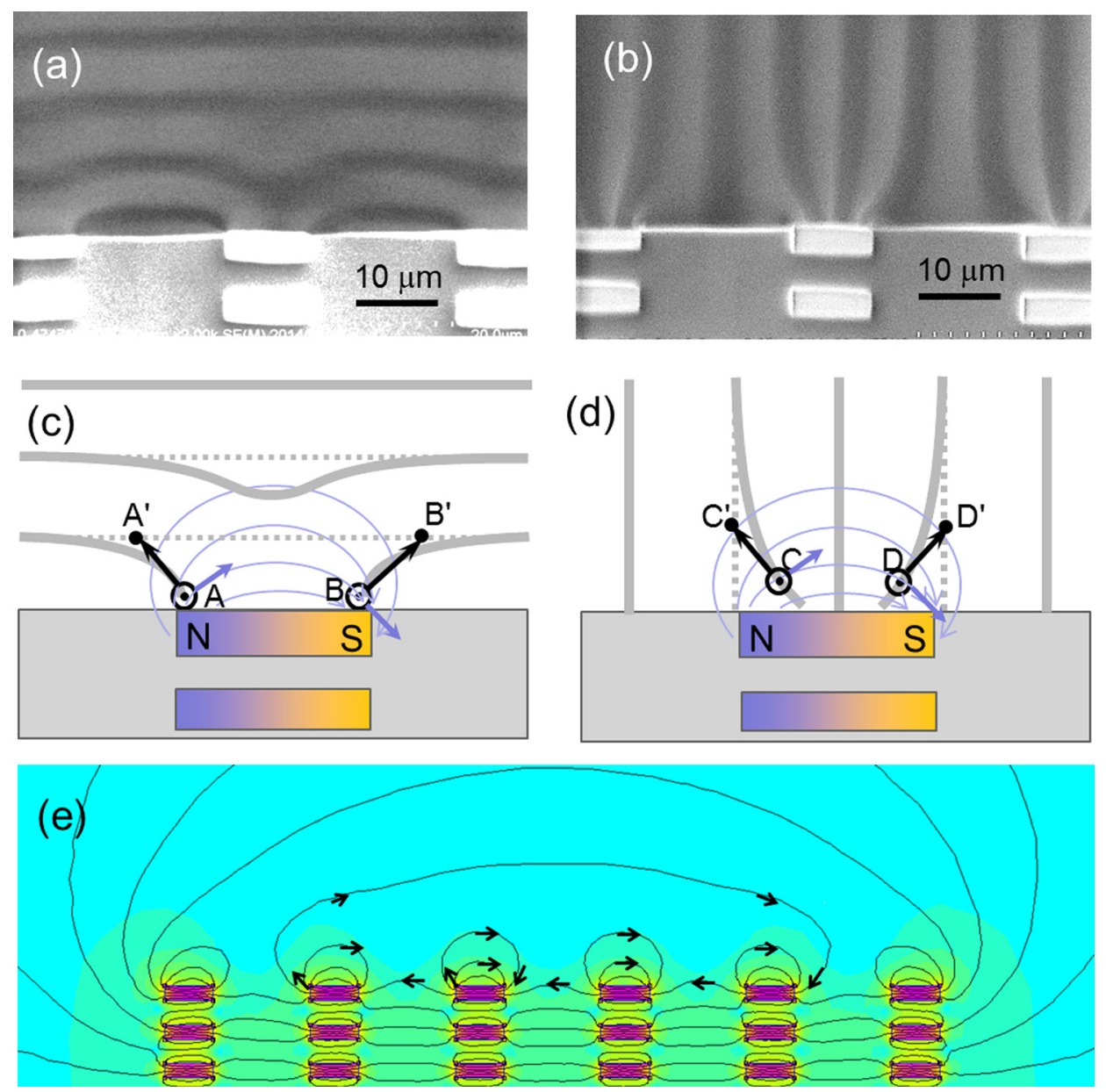

FIG. 2. (Color online) Typical examples of the visualized field images obtained with a 1-keV electron beam and using (a) a horizontal grid and (b) a vertical grid. Illustrations of the corresponding deflection schemes are shown in (c) and (d), respectively. (e) The field distribution for the corresponding Co dot array calculated using the FEM solver platform. 
To evaluate the strength of the local magnetic field, we first consider a simple beam deflection model, as shown in Fig. 3(a). Here, the magnetic material is thin enough to be regarded as a two-dimensional film. Magnetic polarization creates a certain field distribution, but the negligible thickness produces flux lines that are nearly circular in the beam incidence plane. Let us assume now that the field thickness, $l_{z}$, is equivalent to the nearest distance from the material edge. Here, the deflection direction is determined by the Lorentz force, $F_{e}=e \overrightarrow{v_{0}} \times \vec{B}$, and the horizontal velocity after the electron passes through the material can be written as

$$
v_{h}=-\frac{e v_{0} B}{m_{e}}\left(\frac{l_{z}}{v_{0}}\right),
$$

where the transit time in the field is $l_{z} / v_{0}$. The deflection angle $\theta$ can now be defined by $\tan \theta=e B l_{z} / m_{e} v_{0}$, using the electron mass $m_{e}$, the magnetic flux density $B$, the elementary charge $e$, the electron velocity $v_{0}$, and the effective thickness of the magnetic field $l_{z}$. We also define a twodimensional system of coordinates in the SEM image in which the positive $x$-direction is horizontally to the right and positive $y$-direction is vertically upwards.

The grid deformation at point $\mathrm{A}$ is about $4 \mu \mathrm{m}$ from the original grid position indicated by the dashed line in Fig. 2(c), but the exact deviation can be about $5.3 \mu \mathrm{m}$ when we take into account the shrinking ratio of the grid spacing owing to the enlarged scanning area at the grid position. Thus, the deflection angle will be 0.24 mrad. We also assume that the effective field thickness, $l_{z}$, is $4.5 \mu \mathrm{m}$, which is identical to the distance from the edge of the specimen, and this makes the $x$-component of the local magnetic field at point $\mathrm{A}$ to be $55 \mathrm{G}$.

Using the same evaluation procedure for point $\mathrm{B}$, $\sim 3.3 \mu \mathrm{m}$ of grid deformation corresponds to $\sim 0.2 \mathrm{mrad}$ of
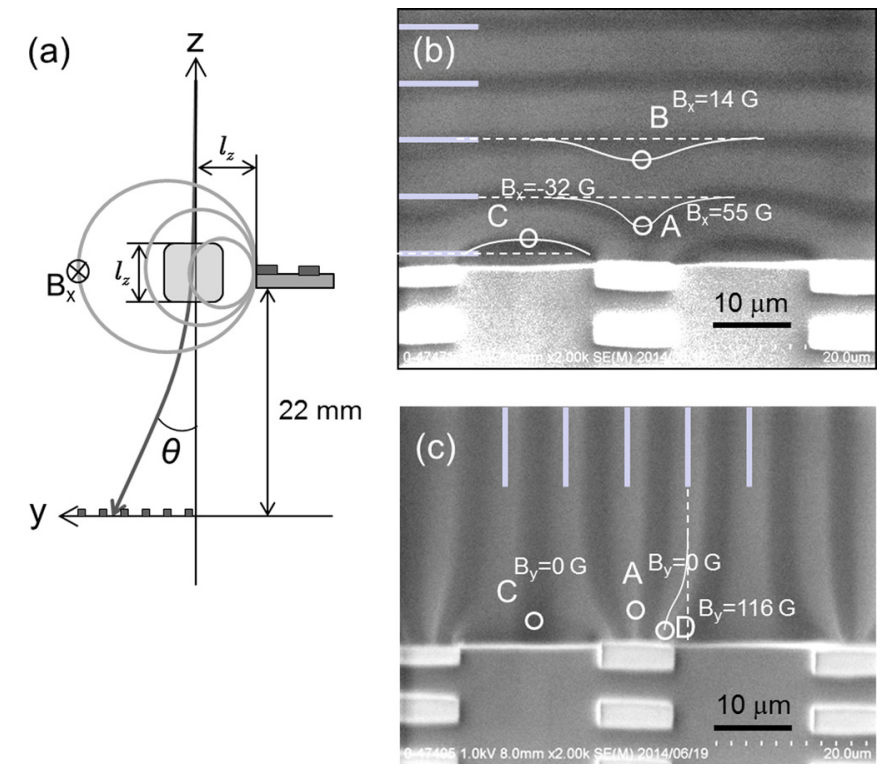

FIG. 3. (Color online) By assuming this (a) cylindrical field distribution in the $y$-z plane, the (b) $x$-component, and (c) $y$-component of the local magnetic field are estimated. deflection angle. Thus, the local magnetic field at point $\mathrm{B}$ is estimated to be $\sim 14 \mathrm{G}$, considering that the nearest distance from the edge of the rectangle is $17 \mu \mathrm{m}$. By contrast, the deformation at point $\mathrm{C}$ is in the negative direction, suggesting that the flux direction is opposite those existing at points A and B. Using the grid deformation of $1.8 \mu \mathrm{m}$ and the distance of $3.5 \mu \mathrm{m}$ from the specimen edge, the local magnetic field $\mathrm{B}_{\mathrm{x}}$ is estimated to be $\sim 32 \mathrm{G}$. This tendency of the flux to be in negative $x$-direction agrees well with the results found using FEM simulation, but the absolute value obtained in these is about three times larger than that obtained with the FEM simulation.

The $y$-component of the magnetic field, $B_{y}$, at points $\mathrm{C}$ and $\mathrm{A}$ is exactly zero and, as shown in Fig. 2(d), the vertical grid lines are not deformed at these points. By contrast, the large deformation at point $\mathrm{D}$ is about $0.17 \mathrm{mrad}$, and the distance of $1 \mu \mathrm{m}$ from the cobalt dot rectangle array edge results in a large local field of 116 G. A similar value is yielded by FEM simulation if we assume a field coercivity of 500 Oe for the cobalt dot rectangular array. ${ }^{16,26}$

Because the deflection angle is inversely proportional to the electron velocity, the deflection characteristics have a sensitive dependence upon the accelerating voltage, as shown in the SEM images in Fig. 4. The convex deformation of the horizontal grid image as well as the vertical grid shadow convergence becomes small as the accelerating voltage increases. Thus, the detection sensitivity of the deflection is remarkably improved at lower accelerating voltages, but there is a tradeoff between the image resolution and this detection sensitivity.

Figure 5 shows an example of collective magnetization that is observed on the stripe array, as shown in Fig. 5(a). Here, all of the grid shadows are somewhat shifted to the right, and the grid shadow deformation is also tilted in the upward right direction [Fig. 5(b)]. By contrast, when the stripe array is magnetized in the opposite direction, the tilting is in the upward left direction [Fig. 5(c)]. Because of the tilting of the shadow lines, the location of the most intense deformation is also shifted to the right side of the stripe in Fig. 5(b). This position shift and tilting can be easily understood if one assumes the tilting of the magnetizing direction, as shown by large gray arrows. The magnetization parallel to the stripe should yield the lowest magnetizing energy for a single cobalt stripe. However, the collective magnetization realized in the square-shaped stripe array strongly deviates from the stable magnetization direction of the stripe, and thereby creates an unbalanced magnetic pole at the top corner of the stripe, as shown in the FEM simulation results in Fig. 5(d). Thus, the deviation of $\sim 30^{\circ}$ from the stripe direction creates the asymmetric deformation of the shadows.

The grid shadows seem to deviate gradually from their original position located $\sim 60 \mu \mathrm{m}$ from the cobalt stripes, as shown by the eye-guide lines. The local magnetic field can be evaluated based on the slight distortion at point A [Fig. 5 (b)], where the deviation at this point is $\sim 1 \mu \mathrm{m}$. Thus, the local magnetic field will be $\sim 0.5 \mathrm{G}$ considering that point $\mathrm{A}$ is $\sim 50 \mu \mathrm{m}$ from the edge, yielding a field resolution that can be useful in evaluating spintronic devices. 

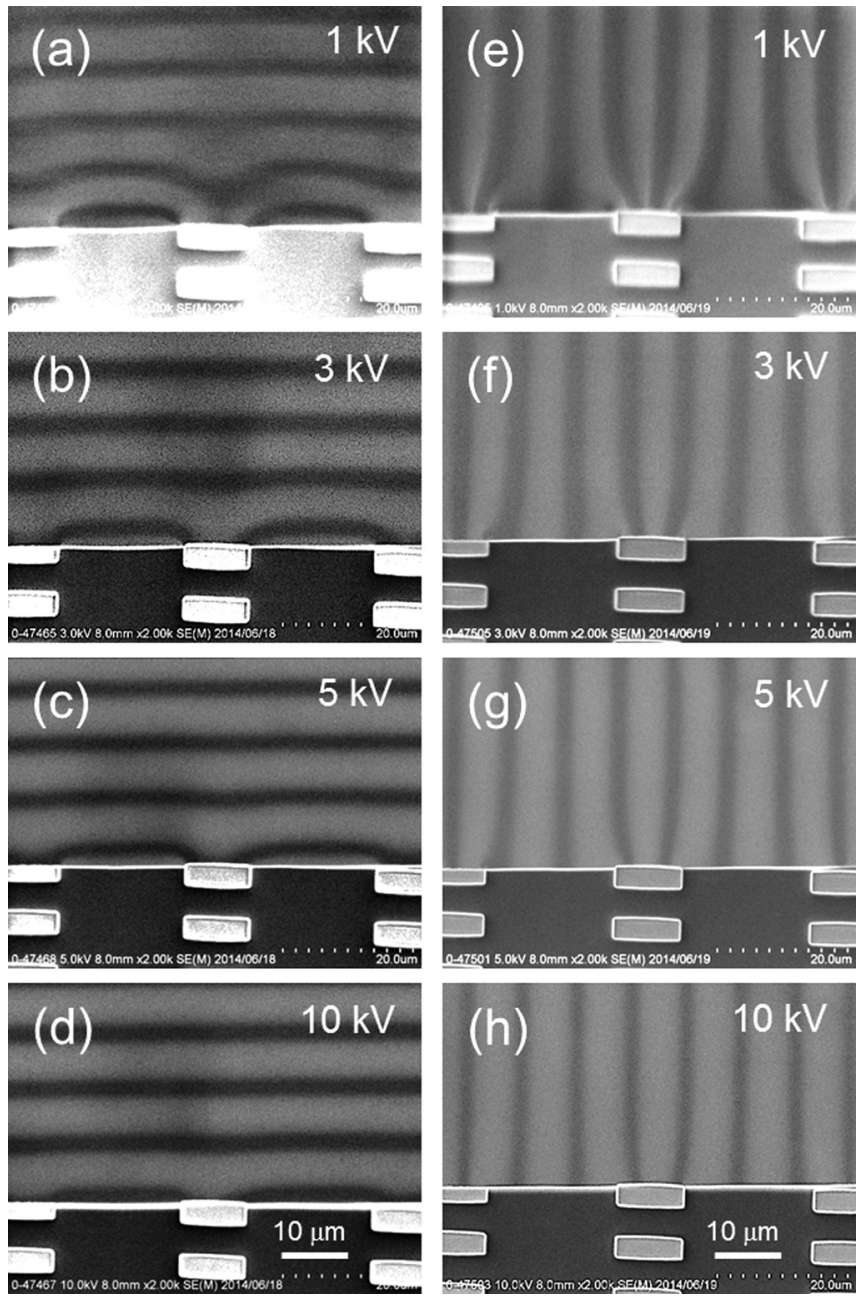

FIG. 4. Deformations of the horizontal grid as a function of the accelerating voltage at (a) 1 , (b) 3, (c) 5, and (d) $10 \mathrm{keV}$. Deformations of the vertical grid as a function of the accelerating voltage at (e) 1 , (f) 3, (g) 5, and (h) $10 \mathrm{keV}$.

\section{DISCUSSION}

This field visualizing technique using electron beams with low acceleration energy combined with horizontal and vertical grids has been clearly demonstrated. Using such visualization, it is possible to resolve field strengths on the order of $0.5 \mathrm{G}$. The principal approximation is carried out using the beam deflection, wherein the deflection angle for the vertical direction is evaluated from the deformation of the horizontal grid, which yields the horizontal field component. However, because the electrons are also deflected in the $y$-direction, the $y$-component of the electron velocity should also impact its motion in the $x$-direction. This indirect deviation $\Delta x$ can be written

$$
\Delta x=\frac{1}{2}\left(\frac{e}{m_{e}} v_{0} B_{x}\right)\left(\frac{l_{z}}{v}\right)^{2}=e B_{x} l_{z}^{2} / 2 m_{e} v_{0} .
$$

The indirect deviation at point $\mathrm{A}$ in Fig. 2(c) is found to be $\Delta x=0.43 \mathrm{~nm}$, which is much smaller than the deflection in $y$-direction and can be ignored when the examined field is relatively weak.
We also assume that the field thickness $l_{z}$ is equivalent to the distance from the cobalt dot rectangular array edge or the edge of the specimen. The model specimen used here, composed of an array of rectangular-shaped dots, can be regarded as a single and uniformly magnetized material when the examined point is at a certain distance from the specimen edge, which is exactly what the FEM simulation shows for the collective flux distribution [Fig. 2(e)], excepting the detailed field distribution near the rectangles themselves. Therefore, it is conceivable that in the proximity of the rectangles the field has a cylindrical distribution and that circular contours appear in the cross section of the $y-z$ plane, as shown in Fig. 3(c). Consequently, the assumption of the effective field thickness is reasonably supported by the field thickness being almost identical to the distance from the edge of the dot array, and the evaluated field strength as well as the flux direction agrees well with the FEM simulation results. Therefore, the local magnetic field characteristics at points A, B, and D in Figs. 2(b) and 2(c) agreed satisfactorily with the FEM simulation results.

However, the evaluated magnetic flux at point $\mathrm{C}$, which is located in the intermittent space between the two dots, is found to be much larger than the flux predicted in the FEM simulation. Here, the magnetic field can be expressed as a vector sum of fluxes from both the divergent and convergent nodes, and therefore, a cylindrical distribution description of the magnetic flux cannot be adopted. If we assume that the effective field thickness at point $\mathrm{C}$ is identical to the distance from the node, the obtained local field intensity will be $\sim 10 \mathrm{G}$, showing better agreement with the FEM simulation results.

The assumption regarding the effective field thickness is based on a cylindrical field distribution generated by a twodimensional magnetic material, which results in a certain evaluation error. The evaluation procedure requires further optimization and improvement. In particular, there is a need to improve evaluation around singular points, such as those at the magnetic pole and around corners, at which the magnetic flux converges or diverges.

\section{SUMMARY}

We demonstrate the magnetic field visualization technique by using the beam deflection of electrons with low acceleration energy in the local magnetic field around a microsized specimen. Combined with horizontal and vertical grids positioned downstream of the specimen, a local magnetic field is projected onto the same SEM image of the specimen on which the deformed shadows of the grid lines are projected. The deformation is strongly correlated with the local magnetic field and is related to the Lorentz force. The deformation of the horizontal grid line represents the horizontal component of the local magnetic field, and the deformation of the vertical grid line corresponds to the vertical component of the local field. The local magnetic field induced by a two-dimensional magnetic material yields a rather simple field distribution, characterized by a cylindrical contour of the magnetic field intensity, which allows for an analytical calculation of the local magnetic field intensity. A detection sensitivity of $\sim 0.5 \mathrm{G}$ is achieved by using a 

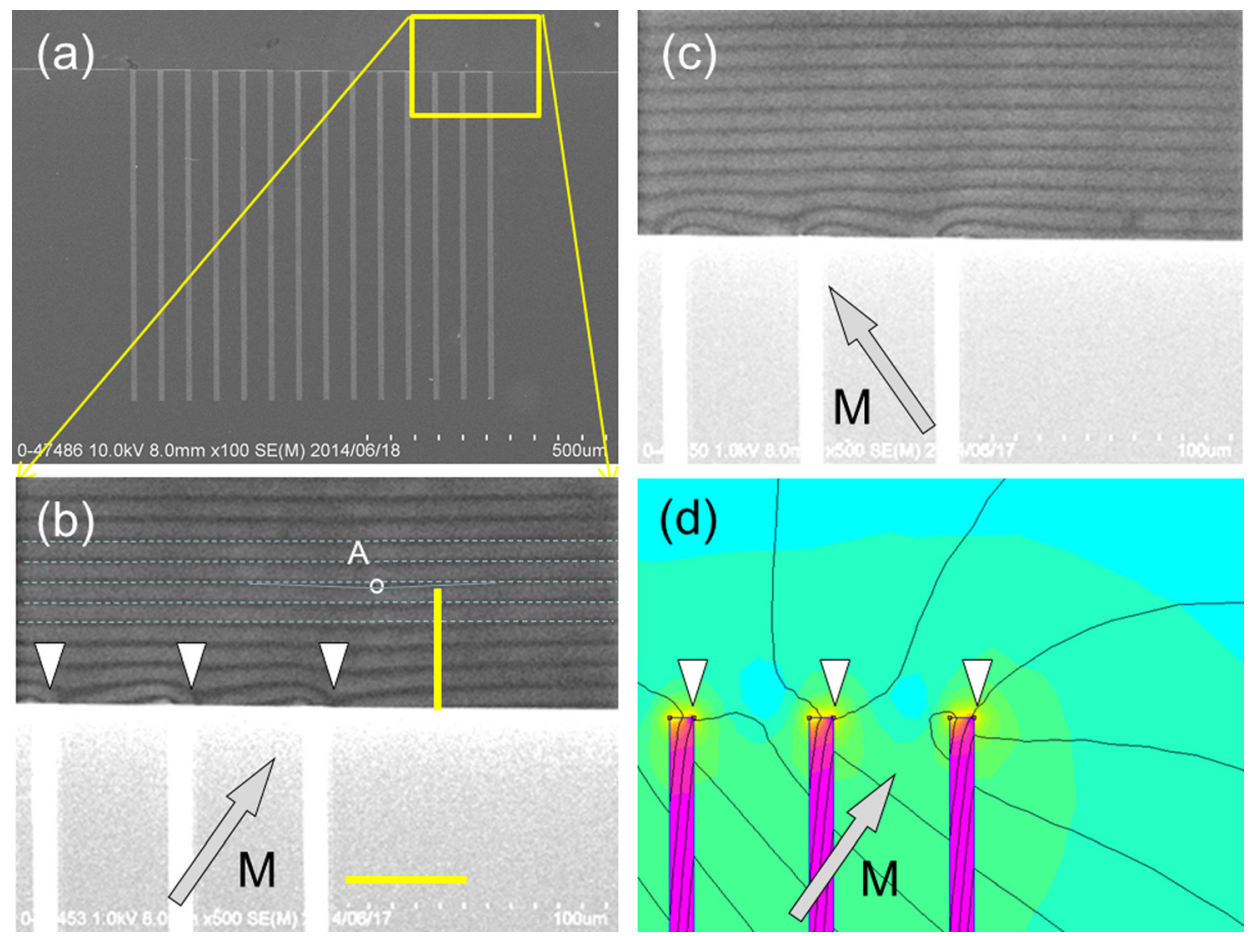

FIG. 5. (Color online) Example of collective magnetization: (a) the entire shape of the stripe array, (b) the local magnetic field image with right-hand tilted magnetization, and (c) the local magnetic field image with left-hand tilted magnetization. (d) The local magnetic field in the top right corner (indicated by the white arrow) of each stripe is larger than that in the left corner in the FEM simulation, in good agreement with the field image in (b).

primary electron beam with an energy of $1 \mathrm{keV}$. The measured field intensity agrees satisfactorily with the FEM simulation results. The analysis method and the detection technique require further improvement. In the meantime, our simple visualization method offers a promising approach for exploring future spintronic devices.

\section{ACKNOWLEDGMENT}

This work was supported by the Japanese Society for the Promotion of Science Grants-in-Aid for Scientific Research (Kakenhi) (Grant No. 26107506).

${ }^{1}$ F. J. Dyson, Phys. Rev. 102, 1217 (1956).

${ }^{2}$ L. D. Faddeev and L. A. Takhtajan, Phys. Lett. A 85, 375 (1981).

${ }^{3}$ J. A. Katine, F. J. Albert, R. A. Buhrman, E. B. Myers, and D. C. Ralph, Phys. Rev. Lett. 84, 3149 (2000).

${ }^{4}$ J. Podbielski, F. Giesen, and D. Grundler, Phys. Rev. Lett. 96, 167207 (2006).

${ }^{5}$ J. Nitta, F. E. Meijer, and H. Takayanagi, Appl. Phys. Lett. 75, 695 (1999).

${ }^{6}$ N. Sato, K. Sekiguchi, and Y. Nozaki, Appl. Phys. Express 6, 063001 (2013).

${ }^{7}$ H. J. Chia, F. Guo, L. M. Belova, and R. D. McMichael, Phys. Rev. Lett. 108, 087206 (2012)

${ }^{8}$ K. Tada, J. Haruyama, H. X. Yang, M. Chshiev, T. Matsui, and H. Fukuyama Phys. Rev. Lett. 107, 217203 (2011).
${ }^{9}$ T. Shimizu, J. Nakamura, K. Tada, Y. Yagi, and J. Haruyama, Appl. Phys. Lett. 100, 023104 (2012).

${ }^{10}$ D. Rugar, R. Budakian, H. J. Mamin, and B. W. Chui, Nature 430, 329 (2004).

${ }^{11}$ L. Zhang, C. Israel, A. Biswas, R. L. Greene, and A. de Lozanne, Science 298, 805 (2002).

${ }^{12}$ D. Gabor, Nature 161, 777 (1948).

${ }^{13}$ G. Möllenstedt and H. Düker, Z. Phys. 145, 377 (1956).

${ }^{14}$ A. Tonomura, Rev. Mod. Phys. 59, 639 (1987).

${ }^{15}$ M. Lehmann and H. Lichte, Microsc. Microanal. 8, 447 (2002).

${ }^{16}$ R. E. Dunin-Borkowski, M. R. McCartney, R. B. Frankel, D. A. Bazylinski, Mi. Pósfai, and P. R. Buseck, Science 282, 1868 (1998).

${ }^{17}$ M. R. McCartney and D. J. Smith, Annu. Rev. Mater. Res. 37, 729 (2007).

${ }^{18}$ R. E. Dunin-Borkowski, T. Kasama, A. Wei, S. L. Tripp, M. J. Hÿtch, E. Snoeck, R. J. Harrison, and A. Putnis, Microsc. Res. Tech. 64, 390 (2004).

${ }^{19}$ H.-W. Fink, W. Stocker, and H. Schmid, Phys. Rev. Lett. 65, 1204 (1990).

${ }^{20}$ P. J. Grundy and R. S. Tebble, Adv. Phys. 17, 153 (1968).

${ }^{21}$ D. Wohlleben, J. Appl. Phys. 38, 3341 (1967).

${ }^{22}$ J. Fujita, Y. Ikeda, and I. Suzuki, Jpn. J. Appl. Phys. 48, 06FG02 (2009).

${ }^{23}$ J. Fujita, Y. Ikeda, and I. Suzuki, J. Vac. Sci. Technol., B 26, 2069 (2008).

${ }^{24}$ J. Fujita, Y. Ikeda, S. Okada, K. Higashi, and S. Nakasawa, J. Vac. Sci. Technol., B 25, 2624 (2007).

${ }^{25}$ J. Fujita, Y. Ikeda, S. Okada, K. Higashi, S. Nakazawa, M. Ishida, and S. Matsui, Jpn. J. Appl. Phys., Part 1 46, 498 (2007).

${ }^{26}$ N. Deo, M. F. Bain, J. H. Montgomery, and H. S. Gamble, J. Mater. Sci.: Mater. Electron. 16, 387 (2005). 